\title{
A implementação das prioridades da Política Nacional de Promoção da Saúde, um balanço, 2006 a 2014
}

\author{
The implementation of the priorities of the National Health \\ Promotion Policy, an assessment, 2006-2014
}

\author{
Deborah Carvalho Malta ${ }^{1,2}$ \\ Marta Maria Alves da Silva ${ }^{1}$ \\ Geórgia Maria Albuquerque ${ }^{1}$ \\ Cheila Maria de Lima ${ }^{1}$ \\ Tania Cavalcante ${ }^{3}$ \\ Patrícia Constante Jaime ${ }^{4,5}$ \\ Jarbas Barbosa da Silva Júnior ${ }^{6}$
}

${ }^{1}$ Departamento de

Vigilância de Doenças e Agravos Não Transmissíveis

e Promoção da Saúde, Secretaria de Vigilância em Saúde, Ministério da Saúde (MS). SAF Sul, Trecho 2, Lote 5/6, Torre I, Edifício Premium Sala 14/térreo. 70070-600 Brasília DF Brasil. deborah.malta@ saude.gov.br

${ }^{2}$ Universidade Federal de Minas Gerais.

${ }^{3}$ Secretaria Executiva da Comissão Nacional para Implementação da Convenção Quadro para Controle do Tabaco Instituto Nacional do Câncer, MS.

${ }^{4}$ Departamento de Atenção Básica, Secretaria de Assistência a Saúde, MS.

${ }^{5}$ Universidade de São Paulo.

${ }^{6}$ Secretaria de Vigilância em

Saúde, MS.

\begin{abstract}
The scope of this article is to analyze the National Health Promotion Policy with respect to the implementation of core management priorities. Information contained in institutional documents, websites, books and published articles was consulted in order to analyze the actions implemented. There were advances in management, such as the creation of a specific budget line, insertion of the promotion in the Multi-Year Plan, monitoring of indicators of health promotion in the federal pacts, financing of health promotion projects in municipalities and the creation of health promotion programs. Evaluation of physical activity programs was conducted that revealed the effectiveness of the programs. Intersectorial actions taken were relevant, in particular coordination with the Education, Justice, Cities, Human Rights, Social Development, Sports and Leisure sectors, among others. Regulatory actions have been implemented such as the Drink and Drive ban and no smoking environments. Advances were observed, especially greater emphasis on health promotion in the health sector agenda as well as partnerships as intersectorial actions, identifying inequities in the area seeking reduction thereof, in addition to the sustainability of health promotion actions.
\end{abstract}

Key words Health, Health promotion, Intersectoriality, Planning, Management, Public policy
Resumo O objetivo deste artigo é analisar a Política Nacional de Promoção da Saúde (PNPS) quanto à implementação de eixos prioritários de gestão. Foram consultadas informações contidas em portarias, documentos institucionais, sites, livros e artigos publicados visando analisar as ações implementadas. Houve avanços na gestão como a criação de linha orçamentária específica, a inserção da promoção no Plano Plurianual, monitoramento de indicadores da Promoção da Saúde nos pactos federativos, ofinanciamento de projetos de Promoção da Saúde em municípios e a criação de programas de Promoção da Saúde. Foram realizadas avaliações de programas de atividade física que apontaram a efetividade dos programas. Ações intersetoriais implementadas foram relevantes, em especial a articulação com os setores de Educação, Justiça, Cidades, Direitos Humanos, Desenvolvimento Social, Esporte e Lazer, dentre outros. Ações regulatórias foram implementadas, como a Lei "Seca" e a lei de ambientes livres de tabaco. Foram observados avanços e pontuamos o fortalecimento da Promoção da Saúde na agenda do setor saúde, aprofundamento nas parcerias $e$ nas ações intersetoriais, a identificação das iniquidades no território visando sua redução, bem como a sustentabilidade das ações de Promoção da Saúde.

Palavras-chave Saúde, Promoção da saúde, Intersetorialidade, Planejamento, Gestão, Políticas públicas 


\section{Introdução}

A Promoção da Saúde tem como referencial teórico a Carta de Ottawa ${ }^{1}$ e a busca da redução das iniquidades em saúde, visando o empoderamento dos indivíduos ou grupos ${ }^{2}$. A Promoção tem como finalidade ampliar as possibilidades de indivíduos e comunidades atuarem sobre fatores que afetam sua saúde e qualidade de vida, com maior participação no controle deste processo ${ }^{3,4}$.

Os princípios da Promoção da Saúde foram incorporados pelo Movimento da Reforma Sanitária na Constituição Federal de 1988 e no Sistema Único de Saúde (SUS), e sua efetiva institucionalização ocorreu em 2006, com a aprovação da Política Nacional de Promoção da Saúde (PNPS) pela Comissão Intergestores Tripartite ${ }^{5,6}$. A publicação da PNPS representa um marco na consolidação do SUS, uma vez que reafirma o debate dos condicionantes e determinantes sociais da saúde no processo saúde-doença. A PNPS estabelece como objetivo promover a qualidade de vida e reduzir vulnerabilidades e riscos à saúde relacionados aos seus determinantes e condicionantes - modos de viver, condições de trabalho, habitação, ambiente, educação, lazer, cultura, acesso a bens e serviços essenciais. Suas diretrizes preconizam atitudes baseadas na cooperação e no respeito às singularidades, como: estímulo à intersetorialidade, compromisso com a integralidade do cuidado, fortalecimento da participação social e estabelecimento de mecanismos de cogestão no processo de trabalho em equipe $e^{5,6}$.

Como forma de operar a transversalidade da PNPS no SUS e construir mecanismos de corresponsabilização e cogestão, foi criado pelo Ministério da Saúde, ainda em 2005, o Comitê Gestor da PNPS, para coordenar as ações de Promoção da Saúde. O Comitê Gestor da PNPS - CGPNPS envolve diferentes secretarias e áreas do Ministério da Saúde, Conselho Nacional de Secretários Estaduais de Saúde (Conass), Conselho Nacional de Secretários Municipais de Saúde (Conasems) e da Associação Brasileira de Saúde Coletiva (Abrasco) ${ }^{7,8}$. Durante estes anos, a atuação do Comitê Gestor da PNPS foi fundamental na coordenação das ações intra e intersetoriais da Promoção da Saúde.

O texto da PNPS aponta como prioridade de ação temas como: alimentação saudável, prática corporal e atividade física, prevenção e controle do tabagismo, redução da morbimortalidade em decorrência do uso abusivo de álcool e outras drogas, redução da morbimortalidade por acidentes de trânsito, prevenção da violência e estímulo à cultura de paz e promoção do desenvolvimento sustentável. Estas ações prioritárias apontadas na PNPS serviram como dispositivo indutor para o fortalecimento de ações de promoção em todos as esferas do SUS $^{5}$ e a melhoria da articulação entre diferentes áreas técnicas e de programas e políticas a partir da abordagem da Promoção da Saúde 5 .

A inclusão destes temas passou pelos critérios do quadro de magnitude da morbimortalidade, transcendência, atuação intersetorial, efetividade das práticas de Promoção da Saúde na resposta aos temas prioritários nos três níveis de gestão $0^{9-14}$.

Existem evidências sobre os benefícios de programas populacionais voltados para o incentivo à atividade física e alimentação saudável em âmbito comunitário e na escola ${ }^{11,14-17}$. As políticas públicas na área de urbanização e ambiente têm grande potencial para reduzir morbimortalidade por acidentes de trânsito, aumentar os níveis de atividade física da população, como por exemplo: mobilidade e espaços urbanos que propiciem a prática de caminhadas, ciclismo, e o transporte ativo de forma acessível e segura. Estudos apontam evidências nos benefícios de programas de promoção de alimentação saudável e atividade física nas escolas ${ }^{12,14}$, além de inúmeras evidências dos benefícios das ações de regulação de ambientes livres de tabaco ${ }^{12}$, proibição da propaganda do fumo ${ }^{12,13}$, legislação relativa à proteção das populações em relação aos limites de álcool e direção ${ }^{11,18}$, restrição à propaganda de alimentos na infância, rotulagem de alimentos ${ }^{12}$, programas de apoio às famílias e proteção das vítimas de violência ${ }^{19,20}$, dentre outros, mostrando a importância destas ações na proteção da população, bem como a articulação intersetorial e a mobilização de parceiros visando respostas mais efetivas e integradas ${ }^{5,12}$.

O objetivo deste artigo é analisar a Política Nacional de Promoção da Saúde (PNPS) quanto à implementação de sua agenda prioritária.

\section{Metodologia}

Trata-se de estudo de revisão que buscou responder o alcance ou não das ações/atividades inscritas na PNPS, quanto aos temas definidos como prioritários em 2006. Foram consultadas informações contidas em portarias do governo Federal entre 2005 a 2013, documentos e publicações institucionais do Ministério da Saúde, consultas ao site do Ministério da Saúde, livros e artigos científicos referenciados no site, relacio- 
nados ao tema da Política de Promoção da Saúde e suas prioridades. Foram ainda realizadas pesquisas bibliográficas na base da Bireme com os descritores Promoção da Saúde, política, Brasil e atividade física, sendo identificados quinze (15) artigos; e, subsequentemente, foram pesquisados os três primeiros descritores e os demais temas prioritários da PNPS, tendo sido identificados em relação à alimentação saudável (14), artigos, prevenção de violência (20), tabagismo (3), trânsito (1) e ambiente sustentável (7). Quando todos os descritores eram pesquisados em conjunto, nenhum artigo foi localizado. Foram excluídos os artigos que tinham abordagem de programas locais, ou que analisaram outros temas da Promoção da Saúde não relacionados às prioridades, cerca de 10 artigos foram analisados, além dos documentos institucionais acima descritos.

Para sistematizar o balanço foram definidos alguns temas transversais que foram tomados como analisadores das diferentes prioridades aqui incluídas como objeto de estudo como: 1) Fortalecimento da Promoção da Saúde no SUS (gestão, financiamento, organização da informação e vigilância; qualificação da força de trabalho, regulação e controle); 2) Ações de mobilização social e divulgação; 3) Articulação intersetorial e parcerias; 4) Promoção de ações no território, 5) Avaliação e monitoramento. Por fim, na discussão, incluímos uma reflexão sobre os desafios futuros na implementação do da Promoção da Saúde.

\section{Resultados}

\section{Fortalecimento da Promoção da Saúde no SUS}

\section{Gestão}

Em 2006 foram dados importantes passos na institucionalização da Política Nacional de Promoção da Saúde no SUS, como a sua aprovação na Comissão Intergestores Tripartite (CIT), a criação de linha de programação orçamentária específica para Promoção da Saúde, com inserção no Plano Plurianual e no Plano Nacional de Saúde ${ }^{6}$.

Entre 2008 e 2011, a PNPS foi incluída na agenda Interfederativa, por meio do Pacto Pela Vida; ocorreram, ainda, a introdução de indicadores de monitoramento, como a redução da prevalência de sedentarismo e tabagismo nas capitais, e a implantação dos núcleos de prevenção de violências e promoção da saúde ${ }^{6}$. A partir de
2011, no Contrato Organizativo de Ação Pública (COAP), também foram inseridos indicadores de Promoção da Saúde, como notificação de violência doméstica, sexual e/ou outras violências, implementação do Programa Academia da Saúde, entre outros.

Dentre os programa de governo destacamse o "Mais Saúde", entre 2008 e 2010, inserindo no monitoramento ações de Promoção da Saúde como as reuniões do Comitê Gestor da PNPS; repasses financeiros para programas de Promoção da Saúde, como os de promoção de atividade física e alimentação saudável, prevenção do tabagismo, implementação de núcleos de prevenção de violência, vigilênciavigilância e prevenção de lesões e mortes no trânsito. Estas ações também foram inseridas em 2011 no Plano Nacional de Saúde (2011-2015) e no Planejamento Estratégico do Ministério da Saúde (2011-2015). Destacando-se a implantação do Programa Academia da Saúde, a expansão do Programa Saúde na Escola e o Projeto Vida no Trânsito ${ }^{21-23}$.

Em 2011, no Plano de Enfrentamento das DCNT (2011-2022), foram priorizados os quatro principais fatores de risco modificáveis (tabagismo, alimentação inadequada, inatividade física, consumo abusivo de bebidas alcoólicas) $)^{15,16}$. Foram ainda estabelecidas metas para monitoramento do Plano, como redução de fatores de risco através de inatividade física, alimentação inadequada, tabaco e álcool, o que apoiou a prioridade da Promoção da Saúde na agenda do SUS ${ }^{15,16}$.

\section{Financiamento}

Desde 2005, o Ministério da Saúde tem financiado entes federados na realização de programas de Promoção da Saúde. Tal iniciativa, iniciada com as capitais dos Estados e Distrito Federal, evoluiu de forma acentuada e, entre 2006 e 2010, foram repassados cerca de R 171 milhões às Secretarias Estaduais e em torno de $1.500 \mathrm{Se}$ cretarias Municipais de Saúde de todas as regiões do país, que integraram a Rede Nacional de Promoção da Saúde. Nestes anos iniciais, 2005 a 2010, foi pactuada na Comissão Intergestora Tripartite (CIT) a estratégia de seleção por meio de editais ou portarias públicas, com envio de projetos pelos municípios, que eram avaliados e selecionados segundo a disponibilidade orçamentária. Com esses recursos, os gestores públicos desenvolveram projetos de Promoção da Saúde, contemplando, em sua maioria, ações de promoção da atividade física, prevenção de violência e cultura da paz, e redução da morbimortalidade por trânsito. Entre 2008 e 2010, também foram 
financiados programas para as demais prioridades da PNPS ${ }^{6,17}$.

A partir de 2011 foram definidas novas modalidades de repasse de recurso, buscando ações continuadas, sustentáveis e universais. No caso da promoção de atividades físicas e práticas corporais, definiu-se pela implantação do Programa Academia da Saúde ${ }^{17,21,22}$, com recursos financeiros provenientes do Piso Variável em Vigilância e Promoção da Saúde (PVPVS), e do Piso de Atenção Básica Variável - PAB Variável - da Secretaria de Atenção da

Saúde ${ }^{21,22}$, para construção dos polos do programa e custeio de suas atividades. Esses recursos se destinam a apoiar a construção de estruturas físicas e custear as ações nesses espaços, bem como ao custeio de iniciativas municipais e estaduais sob gestão do setor saúde, reconhecidas pelo Ministério da Saúde como similares ao Programa Academia da Saúde.

O Programa Saúde na Escola (PSE) foi criado por meio de decreto Presidencial em 2007, e articula ações no ambiente escolar entre a saúde e a educação, e atualmente está implantado em cerca de $87 \%$ dos municípios brasileiros. O PSE apresenta cinco componentes, dentre eles o de Promoção da Saúde e prevenção de agravos, que inclui ações de alimentação saudável, atividade física, prevenção de violência e acidentes, prevenção de DST/AIDS, tabagismo, álcool, dentre outras. Esta ação não estava prevista na primeira versão da PNPS, em 2006, mas passou a ser o principal programa de Promoção da Saúde para o público escolar a partir de 2008. Com a ampliação dos critérios para adesão ao Programa, o PSE passou de 1,9 milhões de educandos beneficiados em 2008, para 18,7 milhões em 2013, com adesão de 4.864 municípios. Para 2014, está previsto o repasse financeiro de $\mathrm{R} \$ 71$ milhões; um aumento de aproximadamente $100 \%$, comparado ao financiamento inicial de $\mathrm{R} \$ 36,5$ milhões.

Em relação aos programas de redução de morbimortalidade no trânsito, a partir de 2006 foram repassados recursos, inicialmente para 16 capitais, e progressivamente expandindo para as demais capitais e cidades com população acima de 1 milhão de habitantes. Em 2010, foi criado o Projeto Vida no Trânsito (PVT) ${ }^{23}$, ampliando parcerias, incluindo novas metodologias para definição e seleção de fatores de riscos, definição dos planos intersetoriais locais, e de grupo intersetorial de condução local e nacional ${ }^{23}$. Assim entre 2006 e 2013 cerca de 50 milhões de reais foram repassados aos municípios. Em 2013, o PVT entrou no Piso Variável de Vigilância e Promoção da Saúde, dando sustentabilidade ao tema e reafirmando os compromissos do SUS de maneira interfederativa ${ }^{24}$.

A Rede Nacional de Prevenção das Violências e Promoção da Saúde foi criada em mais de 1.300 municípios, em conformidade com a Política Nacional de Redução da Morbimortalidade por Acidentes e Violências e visa à atenção integral e proteção às pessoas e suas famílias em situação de violências. Entre 2006 a 2012 foram financiados cerca de 1300 municípios contendo ações de prevenção de violência e acidentes e cultura da paz.

\section{Organização da Informação e da Vigilância}

Visando estruturar informações para apoio à gestão, o Ministério da Saúde tem implementado um sistema contínuo de vigilância de fatores de risco e proteção para Doenças e Agravos Não Transmissíveis (DCNT) e Promoção da Saúde ${ }^{25-29}$. Atualmente, este sistema é composto pelo: 1) Sistema de Vigilância de Fatores de Risco e Proteção para DCNT, Vigitel, que constitui um inquérito telefônico realizado anualmente nas 26 capitais brasileiras e no Distrito Federal ${ }^{26}$; 2) Pesquisa Nacional de Saúde do Escolar (PeNSE) ${ }^{27}$, realizada a cada 3 anos, junto aos escolares do $9^{\circ}$ ano do ensino fundamental do Brasil, já tendo sido realizadas duas edições, em 2009 e 2012; 3) Inquéritos domiciliares a cada 5 anos, como a Pesquisa Nacional de Saúde em 2013, realizada em cerca de 80 mil domicílios, incluindo entrevistas e medidas físicas como aferição de pressão arterial, peso, altura e circunferência abdominal e, em uma subamostra a coleta de material biológico (sangue e urina) ${ }^{28}$; 4) Vigilância de Violências e Acidentes (VIVA), em seus dois componentes: inquéritos periódicos em Unidades de Urgências e Emergências, com o objetivo de identificar o perfil das violências nos hospitais de urgências localizados nos municípios selecionados; e a vigilância contínua de violência, que capta dados de violência doméstica, sexual e/ou outras violências em serviços de saúde, sendo universalizada em território nacional em 2011, por meio de módulo do Sistema de Informação de Agravos de Notificação (Sinan), a partir de $2009^{29}$; 5) Pesquisa de Orçamentos Familiares (Pof), iniciada no final da década de 80 e inclui medidas antropométricas, inquérito sobre consumo alimentar, sendo um importante instrumento para monitoramento do estado nutricional e do consumo alimentar da população brasileira ${ }^{25}$. Os dados de 2008 foram fundamentais para o monitoramento da políticas de alimentação e nutrição; 6) Sistema de Informações de Vigilância Alimentar 
e Nutricional (Sisvan), no acompanhamento das condicionalidades do bolsa família.

\section{Qualificação da Força de Trabalho}

Nestes oito anos da PNPS foram organizados vários seminários, debates, reuniões técnicas, capacitações sobre os diferentes temas, visando ampliar a compreensão do tema da promoção da saúde no SUS. Dentre os eventos, destaca-se o I, II e III Seminário Nacional sobre a Política Nacional de Promoção da Saúde, em 2007, 2010 e 2014, respectivamente, com a participação de profissionais e gestores do SUS de estados e municípios, e representantes de instituições de ensino e pesquisa.

A estratégia de realização de cursos presenciais para a qualificação da força de trabalho do SUS se mostrou insuficiente e foram organizados cursos à distância que têm cumprido o papel de ofertar conteúdos que possam apoiar a qualificação de gestores e profissionais de saúde no tema da Promoção da Saúde. Nos últimos anos, foram realizados cinco edições do Curso de Educação à Distância (EAD) em Promoção da Saúde, uma Parceria com a Universidade de Brasília ${ }^{30}$ e cinco edições do curso de capacitação de prevenção de violências e acidentes em parceria com Centro Latino Americano de Estudos sobre Violência e Saúde Jorge Careli/Claves, da Fundação Oswaldo Cruz para o desenvolvimento do curso a distância Impactos da Violência na Saúde. Foram ofertadas cerca de 4.000 vagas, em média 400 por edição, nestas dez edições de cursos de EAD. Além disto, foram disponibilizados livros, materiais educativos em sites, $\operatorname{artigos}^{31}$.

Outro exemplo de material educativo para formação dos profissionais em saúde e também para o processo de comunicação social e de difusão de informação para a população, consiste no Guia Alimentar para a População Brasileira. Em sua versão de 2006, consistiu nas primeiras diretrizes oficiais para a promoção da alimentação adequada e saudável, sendo um instrumento estratégico de educação alimentar e nutricional. Diante da necessidade de revisão periódica de seu conteúdo, a versão atualizada, a ser lançada em 2014, traz novos paradigmas sobre a necessidade de compreensão das práticas alimentares no contexto do sistema alimentar e de forma coerente com atual estágio da transição nutricional ${ }^{32}$. O processo de reestruturação teve início em 2011 com a realização da $1^{\text {a }}$ Oficina de escuta para orientar a revisão, e em agosto de 2013 aconteceu a 2a Oficina de avaliação técnica de seu conteúdo. Com o intuito de garantir transparência e ampla participação social no processo de revisão, a nova versão do Guia Alimentar ficou disponível para consulta pública durante 90 dias em $2014^{32}$.

Foram realizadas também oficinas estaduais com profissionais de saúde que atuam na rede e gestores, além de profissionais de outros setores e representantes da sociedade civil, e tiveram como objetivos apresentar a nova versão do documento e fomentar a discussão, garantindo um processo amplo, participativo e intersetorial de construção de seu conteúdo.

\section{Regulação e controle}

Medidas regulatórias podem ser efetivas para o enfrentamento da elevação das mortes e ferimentos no trânsito e, por isso, o Ministério da Saúde vem assumindo o protagonismo nas articulações e advocacy em relação à aprovação de Leis que restringem o consumo de bebidas alcoólicas para motoristas, como a Lei ${ }^{\circ} 11.705 / 2008$, popularmente conhecida como "Lei Seca", e a Lei 12.760/2012, que fortaleceu as ações do agente de trânsito na aplicação de medidas que favoreçam a proteção à vida e a prevenção de acidentes de trânsito em relação ao fator álcool e direção veicular. Além disto, destaca-se o aumento do controle e fiscalização no fornecimento de bebidas para menores de dezoito anos.

A política de regulação dos produtos do tabaco é considerada das mais avançadas do mundo, e já desde a década de oitenta avanços na regulação dos ambientes livres, proibição da propaganda do cigarro, introdução das mensagens de advertência nos maços. Em dezembro de 2011, foi sancionada a Lei $n^{\circ} 12.546$, que pró́be o ato de fumar em recintos coletivos, aumenta a taxação dos cigarros para 85\% e define preço mínimo para a venda do cigarro, visando o enfrentamento da venda de produtos contrabandeados. A Lei também aumentou o espaço de advertências dos cigarros, sendo $100 \%$ em uma das faces frontais, $100 \%$ em uma das faces laterais, e ampliou até 2015 para mais 30\% na outra face frontal. A regulamentação dessa lei se deu pelo Decreto da Presidência da República no 8.262 , de 31 de maio de 2014, que estendeu a sua abrangência para ambientes parcialmente fechados por uma parede, divisórias, teto ou até toldo. O Decreto também definiu como responsáveis pela fiscalização as vigilâncias sanitárias de estados e municípios, assim como as penalidades para a infração ${ }^{33}$. A regulamentação do preço mínimo do cigarro foi realizada pelo Decreto 7.555/2011. Ainda em 2011, a Anvisa proibiu o uso de aditivos nos cigarros, mas esta medida está com efeito suspen- 
sivo pelo Supremo Tribunal Federal. Em agosto 2012, a Anvisa abriu o Laboratório de Toxicologia do Tabaco para apoiar as medidas de fiscalização do setor ${ }^{34}$. Em abril de 2013, o Ministério da Saúde publicou a Portaria 571, sobre a ampliação do tratamento dos tabagistas nas unidades do SUS, inclusive com o acesso aos medicamentos e acompanhamento $^{34}$.

A regulação da publicidade de alimentos foi regulada em RDC n 24/2010, pela Agência de Vigilância Sanitária (Anvisa), sendo posteriormente suspensa pelo Poder Judiciário. A retomada desta discussão, principalmente para crianças e jovens, se deu em 2013, no âmbito do Comitê Gestor da PNPS, com a criação de um Grupo de Trabalho no âmbito do Comitê Gestor da PNPS, bem como de um GT interno ao Conselho Nacional de Segurança Alimentar e Nutricional (Consea).

Ao final de 2013, a Anvisa discutiu a revogação/revisão da RDC n ${ }^{\circ}$ 24/2010, como parte da agenda regulatória 2013/2014. Os próximos encaminhamentos serão definidos a partir de um chamamento público proposto para que os interessados se manifestem à respeito do futuro dessas RDC. As alternativas propostas são a elaboração de um Projeto de Lei (PL) de inciativa do Poder Executivo, a inserção de um dispositivo em Medida Provisória ou o investimento em modificações da própria RDC.

Concomitantemente, continua o acompanhamento do trâmite dos Projetos de Lei que tratam de regulação da publicidade de alimentos no Congresso Nacional. Importante vitória alcançada em 2014 foi a resolução do Conselho Nacional dos Direitos da Criança e do Adolescente, ligado à Secretaria de Direitos Humanos da Presidência da República, que aponta como abusiva a publicidade que incentive a criança a consumir determinado produto ou serviço fazendo uso de linguagem, efeitos ou brindes com apelo.

\section{Ações de mobilização social e divulgação}

Um balanço inicial das ações de comunicação e mobilização aponta que foram utilizadas em especial formas de comunicação alternativas, como redes sociais, com destaque para as mídias espontâneas, organizações de sites e mobilização comunitária.

A divulgação dos resultados dos inquéritos (Vigitel, Viva, PeNSE, PNAD) foi feita nas rádios, na $\mathrm{TV}$, em jornais e revistas, dando visibilidade às informações sobre Promoção da Saúde e prevenção de doenças crônicas e acidentes e violências, podendo contribuir para ações de mobilização social. Alguns dos dados divulgados referem-se, por exemplo, ao aumento da obesidade no país e a programas para incentivar a prática da atividade física ${ }^{17}$ e alimentação saudável ${ }^{34,35}$ aos acordos para redução do sal ${ }^{36}$, divulgação sobre dados de violência e acidentes de trânsito, dados sobre a redução da prevalência do tabagismo e a ações de ambientes livres do tabaco.

Uma das ações contínuas foi a celebração dos dias Mundiais da Atividade Física e da Saúde, anualmente, sempre na primeira semana de abril. Foram vários lemas como: Pratique Saúde (2007); Entre para o time onde a saúde e o meio ambiente jogam juntos (2008), articulando o tema da intersetorialidade; Praticar atividade física é tão simples que você faz brincando (2009), Saúde e Qualidade de Vida (2010); Viver com Saúde é uma Grande Vitória (2013), Quem busca Qualidade de Vida Não pode ficar parado (2014), estes últimos chamando atenção para os grandes eventos esportivos, a importância da atividade física na qualidade de vida; dentre outros temas.

\section{Articulação intersetorial e parcerias}

A promoção da saúde deve articular o conjunto das políticas públicas visando influenciar na melhoria da qualidade de vida ${ }^{3}$. A intersetorialidade consiste em processos sistemáticos de articulações, atuações, planejamento e cooperação entre as políticas públicas e os diferentes setores da sociedade. Constitui-se em uma estratégia, articulada entre saberes e práticas, que busca a convergência de recursos humanos, financeiros, políticos e organizacionais ${ }^{37}$.

No âmbito da Promoção da Saúde, a articulação intersetorial tem como objetivo promover a gestão compartilhada entre usuários, movimentos sociais, trabalhadores do setor sanitário e de outros setores, produzindo autonomia e corresponsabilidades ${ }^{5}$. As ações intersetoriais avançam em relação à cultura institucional pautada por ações isoladas, setorializadas, procurado harmonizá-los com as diversas políticas que promovem o avanço na efetivação dos princípios do SUS e na inclusão e justiça social, repercutindo nas diversas políticas no âmbito da saúde e setores afins.

No que se refere às ações intersetoriais de prevenção de violência destacam-se marcos legais como: Plano de Ação para o Enfretamento da Violência Contra a Pessoa Idosa (2005); Política Nacional de Enfrentamento ao Tráfico de Pessoas (Decreto no 5.948, de 26/10/2006); Lei Maria da Penha (Lei no 11.340, de 7/8/2006), Po- 
lítica Nacional de Saúde Integral da População Negra (Portaria MS/GM nº 992, de 13/5/2009); Política Nacional de Saúde Integral de Lésbicas, Gays, Bissexuais, Travestis e Transexuais - LGBT (2008), Política de Atenção Integral à Saúde da Mulher, Linha de Cuidado para a Atenção à Saúde de Crianças, Adolescentes e suas Famílias em Situação de Violências, entre outros.

O Projeto Vida no Trânsito conta com ampla parceria envolvendo instituições nacionais, internacionais e locais, na laboração de um plano de ações integradas e intersetoriais de segurança no trânsito, visando reduzir este quadro de morbimortalidade. Foi implantado em 2010, em cinco capitais brasileiras: Belo Horizonte, Curitiba, Teresina, Palmas, Campo Grande, e, após 2012, expandido para todas as capitais e cidades acima de 1 milhão de habitantes. O PVT utiliza como ferramenta as informações obtidas a partir das análises feitas pelas Comissões Locais de Dados, que orientam as intervenções integradas e intersetoriais nos territórios de maior necessidade. São articuladas ações integradas de educação, engenharia de trânsito, fiscalização, pesquisa e comunicação social. As intervenções são definidas em parceria e as ações executadas segundo as responsabilidades e especificidades de cada instituição. São exemplos as fiscalizações, como as blitzes, para checagem de consumo de álcool em condutores, em que o planejamento e execução se dá de forma integrada entre a Polícia Militar, Órgão Municipal de Trânsito, Polícia Rodoviária Estadual, Polícia Rodoviária Federal, Detran, o que potencializa o impacto da operação ${ }^{23}$.

$\mathrm{Na}$ área de desenvolvimento sustentável foram construídas diversas parcerias com Ministérios do Meio Ambiente, Integração, Cidades, Casa Civil, Secretarias Estaduais de Saúde e Secretarias Municipais de Saúde, para a realização de Planos de Desenvolvimento Sustentável em áreas como a Região Turística do Meio Norte (Piauí, Maranhão e Ceará); Plano de Desenvolvimento Regional Sustentável do Xingu, dentre outros. Cita-se ainda a criação da Câmara Técnica de Saúde em Apoio a Política Nacional de Desenvolvimento Regional (Portaria MS No 1.987, de 12 de setembro de 2012), a Criação do Grupo Técnico Saúde e Licenciamento Ambiental, Portaria GM no2241 de 25 de setembro de 2009, bem como o desenvolvimento de Programa Cidades Sustentáveis - Brasil + 20 - políticas públicas voltadas à gestão ambiental urbana.

No que se refere à governança da Política Nacional de Controle do Tabaco, a Comissão Nacional de Implementação da Convenção-Quadro para Controle do Tabaco (CONICQ), criada por Decreto Presidencial ${ }^{38}$, tem caráter interministerial e representa o fórum governamental responsável por implementar as medidas da CQCT no país. Esta Comissão é integrada hoje por 18 áreas do governo. A presidência da CONICQ cabe ao Ministro da Saúde, e o INCA é responsável pela sua Secretaria-Executiva. Para maiores detalhes, acessar o Observatório da Política Nacional de Controle do Tabaco.

Destacamos as parcerias com o Ministério da Educação, na implementação de ações do Programa Saúde na Escola, e com o Ministério do Esporte, apoiando o Programa Academia da Saúde.

\section{Promoção da Saúde no território}

Dentre as ações no território, destacamos a Rede Nacional de Prevenção das Violências e Promoção da Saúde, que conta com rede capilarizada e que tem avançado na identificação e notificação das violências no território e integrado com as ações intersetoriais na rede de atenção e de proteção às vítimas de violências. Destacamos ainda o Projeto Vida no Trânsito, sua articulação intersetorial, bem como a integração das redes de urgência e emergência do território, na produção do cuidado e na redução da morbimortalidade no trânsito.

No território escolar, o Programa Saúde na Escola se configura como importante espaço para articulação das equipes de atenção básica com os profissionais de educação, proporcionando a sustentabilidade das ações a partir da conformação de redes de responsabilidade compartilhada sobre o território. As ações de Promoção da Saúde e prevenção de doenças e agravos realizadas no PSE visam garantir oportunidade a todos os educandos de fazerem escolhas mais saudáveis e de serem protagonistas do processo de produção da própria saúde. O PSE procura fomentar uma gestão coletiva e participativa das ações, buscando garantir educação e saúde integrais, a partir da participação dos profissionais, dos educandos e da comunidade.

Destacamos ainda ações relativas à alimentação saudável e a articulação com as redes de atenção do território e a produção do cuidado, previstas tanto na PNAN, quanto na PNPS. A organização dessas ações deve considerar as necessidades de cada território, a partir da articulação transversal dos diversos equipamentos sociais disponíveis e buscando a autonomia dos indivíduos e das comunidades, de forma ampla e intersetorial ${ }^{5}$. 
O Programa Academia da Saúde constitui um modelo de intervenção nacional em Promoção da Saúde, visando contribuir para a equidade no acesso a ações voltadas à produção do cuidado e modos de vida saudáveis em espaços qualificados, constituindo-se equipamentos da atenção básica em saúde. O Programa Academia da Saúde tem as práticas corporais e atividade física como um eixo central de suas ações, e também incluindo as demais ações de Promoção da Saúde no seu escopo, como alimentação saudável, prevenção de violência, prevenção de tabaco, álcool e drogas e outras ${ }^{21}$.

\section{Avaliação e monitoramento}

A avaliação é conceituada como Processo de determinação, sistemática e objetiva, da relevância, efetividade, eficiência e impacto de atividades fundamentadas em seus objetivos. É um processo organizacional para implementação de atividades e para colaborar no planejamento, programação e tomada de decisã $0^{37}$. O processo de avaliação dos programas e estratégias implementadas é essencial para se determinar a efetividade das intervenções sobre a saúde da população e sua adequação quanto a acesso da população alvo, qualidade das ações executadas, entre outros ${ }^{37,39}$.

Partindo-se deste pressuposto, o Ministério da Saúde tem adotado diversas medidas com o objetivo de avaliar a efetividade das ações de Promoção da Saúde, dentre elas destacamos:

a) A avaliação do Projeto Vida no Trânsito em cinco cidades, piloto do projeto, apontou elevado percentual de cumprimento das metas de desempenho dos dois programas; aumento da fiscalização de velocidade; aumento da realização de blitzes de checagem de álcool, com aumento do número de testes e redução do percentual de positividade dos mesmos; redução das taxas de mortalidade por 100 mil habitantes em Palmas, Teresina, Belo Horizonte e redução da razão por 10 mil veículos nas cinco capitais; tendência de redução dos riscos de morte nas capitais de maior magnitude que nos seus respectivos estados ${ }^{23}$.

b) Avaliações dos programas de prática de atividade física existentes nos municípios do Brasil, como Recife, Curitiba, Belo Horizonte, Aracaju e Vitória; e na América Latina, por meio do Projeto Guia Útil de Avaliação em Atividade Física (Guia), que conta com a participação e parcerias entre o Ministério da Saúde do Brasil, Organização Pan Americana de Saúde, Centro de Controle e Prevenção de Doenças dos Estados Unidos (CDC), Centro de Pesquisa e Prevenção de Saint Louis, além de universidades nacionais, como a Universidade Federal de Pelotas, Universidade Federal de São Paulo, a Pontifícia Universidade Católica do Paraná, a Universidade Federal de Minas Gerais, e a Universidade Federal de Sergipe ${ }^{40,41}$.

Para a realização do Projeto GUIA de avaliação dos programas de atividade física foram adotadas diferentes estratégias metodológicas, como: análise histórica da implantação dos programas em nível local, identificação do modelo lógico de cada programa, observação sistemática das atividades realizadas no espaço urbano, coleta de dados qualitativos dos profissionais e gestores envolvidos, estudos quantitativos junto aos usuários, bem como realização de estudos por inquérito telefônico e domiciliar ${ }^{40-42}$.

Dentre os principais resultados, estas avaliações e monitoramentos demonstraram intervenções, que, como projetos comunitários, incluem atividade física; políticas públicas e planejamentos que envolvam a comunidade e prática de atividade física na escola são estratégias eficientes para o incremento dos níveis de atividades físicas populacionais ${ }^{40-42}$.

\section{Discussão}

Nestes oito anos desde a sua publicação, a PNPS apresentou diversos avanços e importantes desafios. A agenda de prioridades 2006 a 2007 acabou por ser cumprida, mas também foram inseridas na prática novas ações, que não constavam no texto original. Na que se refere à gestão, foram financiados projetos de promoção da saúde em municípios, criação de programas de Promoção da Saúde como: Vida no Trânsito, Academia da Saúde, Núcleos de Prevenção de Violências e Promoção da Saúde, Saúde na Escola, dentre outros. Foram realizadas avaliação de programas de atividade física e do programa Academia da Saúde, que mostraram sua efetividade. Ações intersetoriais foram relevantes, em especial a articulação com os setores de Educação, Justiça, Cidades, Direitos Humanos, Desenvolvimento Social, Esporte/Lazer, dentre outros. Ações regulatórias foram implementadas, com destaque para a Lei "Seca" e lei de ambientes livres de tabaco. A prevalência do tabagismo tem reduzido de maneira expressiva nos últimos oito anos.

Nos últimos anos, foram assumidas novas demandas e compromissos nacionais, como as agendas sociais coordenadas pela Casa Civil da Presidência da República, como o programa de 
enfrentamento da pobreza, segurança viária, dentre outros; agendas internacionais como a Conferência de Alto Nível ONU - DCNT (2011), a Conferência Mundial dos Determinantes Sociais da Saúde (2011), a Conferência Rio + 20 (2012), a

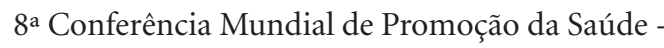
Saúde em todas Políticas (Finlândia, 2013), dentre outras. Assim, o Ministério da Saúde optou pela revisão ampla, participativa e inclusiva da PNPS, quanto aos seus objetivos, diretrizes e ações prioritárias. Nesta revisão, a Abrasco participou de forma ativa na coordenação técnica e metodológica da revisão, visando garantir a ampla participação de diferentes setores. Cabe ressaltar, que nesse amplo processo participativo, a maioria das ações foram mantidas como prioritárias.

A Promoção da Saúde, entendida como estratégia de produção social de saúde, deve articular o conjunto das políticas públicas que influenciem o futuro da qualidade de vida urbana. Assim, as políticas públicas devem considerar a como seu objeto fundamental de atuação a melhoria nas condições de vida, de trabalho, da cultura, estabelecendo uma relação harmoniosa com o meio ambiente, fomentando a participação social ${ }^{3}$. Segundo Moretti et al. ${ }^{3}$ a Promoção da Saúde envolve arranjos intersetoriais na gestão pública, o empoderamento da população, o desenvolvimento de competências e habilidades, capacitação, acesso à informação, estímulo à cidadania ativa, entre outros, para que a população reconheça seus problemas e suas causas, a fim de que ela possa advogar por políticas públicas saudáveis.
Constitui-se um desafio a necessidade de avançar na ação intersetorial, buscando articular ações destinadas a públicos específicos como a Promoção da Saúde no ambiente do trabalho, na comunidade, buscando avançar em projetos destinados à melhoria da mobilidade urbana e na inclusão de pessoas com deficiência e idosos.

Outro desafio consiste em avançar nas ações intersetoriais referentes ao planejamento urbano, remodelação do espaço físico urbano, e definição de políticas públicas voltadas para o ambiente urbano. Estas ações tem grande impacto nos níveis de atividade física populacionais, visando à construção de espaços urbanos saudáveis e que visem à redução de iniquidades sociais, possibilitando o acesso a espaços seguros e saudáveis pela população de baixa renda. Estas ações cabem a diversos setores, incluindo o Ministério das Cidades, Ministério do Transporte, Planejamento Urbano, dentre outros.

Destacamos avanços na inserção de programas de Promoção da Saúde na programação orçamentária; financiamentos destinados aos municípios e estados para projetos de atividade física e práticas corporais; avanços expressivos na vigilância da morbimortalidade e dos fatores de risco e proteção das DCNT; e avanços na avaliação dos projetos, nas parcerias, na capacitação de recursos humanos e na mobilização social. O campo da promoção está em processo de construção e ainda temos um grande caminho a percorrer, mas estão dados os passos para a sua institucionalização e fortalecimento.

\section{Colaboradores}

DC Malta, MMA Silva, GM Albuquerque, CM Lima, T Cavalcante, PC Jaime e JB Silva Júnior participaram igualmente de todas as etapas de elaboração do artigo. 


\section{Referências}

1. World Health Organization (WHO). The Ottawa Charter for Health Promotion. Geneva: WHO; 1986.

2. Buss PM. Uma introdução ao conceito de promoção da saúde. In: Czeresina D, Freitas CM, organizadores. Promoção da saúde: conceitos, reflexões, tendências. Rio de Janeiro: Fiocruz; 2009. p. 12-49.

3. Moretti AC, Almeida V, Westphal MF, Bógus CM. Práticas corporais/atividade física e políticas públicas de promoção da saúde. Saude Soc 2009; 18(2):346-354.

4. World Health Organization (WHO). Milestones in Health Promotion Statements from Global Conferences. Gevena: WHO; 2009.

5. Brasil. Ministério da Saúde (MS). Secretaria de Vigilância em Saúde. Política Nacional de Promoção da Saúde. Portaria n 687 MS/GM, de 30 de março de 2006. Brasília: MS; 2006. (Série B. Textos Básicos em Saúde)

6. Malta DC, Castro AM. Avanços e resultados na implementação da Política Nacional de Promoção da Saúde. Boletim técnico do SENAC 2009; 35(2):63-71.

7. Brasil. Ministério da Saúde (MS). Portaria do Gabinete do Ministro $n^{\circ} 1.409$, de 13 de junho de 2007. Institui Comitê Gestor da Política Nacional de Promoção da Saúde. Diário Oficial da União 2007; 15 jun.

8. Brasil. Ministério da Saúde (MS). Portaria 1.571, de 19 de julho de 2012. Altera a Portaria no 1.409/GM/MS, de 13 de junho de 2007, que institui Comitê Gestor da Política Nacional de Promoção da Saúde. Diário Oficial da União 2012; 23 jul.

9. World Health Organization (WHO). Preventing Cronic Diseases a vital investment. Geneva: WHO; 2005.

10. World Health Organization (WHO). WHO global strategy on diet, physical activity and health. Food Nutr Bull 2004; 25(3):292-302.

11. World Health Organization (WHO). Global Status Report on Alcohol and Health. Geneva: WHO; 2010.

12. World Health Organization (WHO). Global status report on noncommunicable diseases 2010. Geneva: WHO; 2011.

13. Malta DC, Iser BPM, Sá NNB, Yokota RTC, Moura L, Claro RM, Luz MG, Bernal RI. Trends in tobacco consumption from 2006 to 2011 in Brazilian capitals according to the VIGITEL survey. Cad Saude Publica 2013; 29(4):812-822.

14. Brasil. Ministério da Saúde (MS). Secretaria de Vigilância em Saúde. Avaliação de efetividade de programas de atividade física no Brasil. Brasília: MS: 2011.

15. Brasil. Ministério da Saúde (MS). Secretaria de Vigilância em Saúde. Plano de ações estratégicas para enfrentamento das doenças crônicas não transmissiveis (DCNT) no Brasil 2011-2022. Brasília: MS; 2011.

16. Malta DC, Silva Júnior JB. O plano de ações estratégicas para o enfrentamento das doenças crônicas não transmissíveis no Brasil e a definição das metas globais para o enfrentamento dessas doenças até 2025: uma revisão. Epidemiologia e Serviços de Saúde 2013; 22(1):151-164.

17. Malta DC, Silva Júnior JB. Policies to promote physical activity in Brazil. The Lancet 2012; 380(9838):195-196.

18. World Health Organization. Global status report on road safety: time for action. Switzerland: World Health Organization Press; 2009.
19. World Health Organization (WHO). World report on violence and health: summary. Geneva: World Health Organization Press, 2002. [acessado 2013 jan 26]. Disponível em: http://www.who.int/violence_injury_prevention/violence/world_report/en/

20. Mascarenhas MDM, Malta DC, Silva MMA, et al. Violência contra a criança: revelando o perfil dos atendimentos em serviços de emergência, Brasil, 2006 e 2007. Cad Saude Publica. 2010; 26(2):347-357.

21. Brasil. Portaria no 719, de 7 de abril de 2011. Institui o Programa Academia da Saúde no âmbito do Sistema Único de Saúde. Diário Oficial da União 2011; 8 abr.

22. Brasil. Ministério da Saúde (MS). Portaria 2.684, de 8 de novembro de 2013. Redefine as regras e os critérios referentes aos incentivos financeiros de investimento para construção de polos e de custeio no âmbito do Programa Academia da Saúde e os critérios de similaridade entre Programas em desenvolvimento no Distrito Federal ou no Município e o Programa Academia da Saúde. Diário Oficial da União 2013; 14 nov.

23. Morais Neto OL, Silva MMA, Lima CM, Malta DC, Silva Júnior JB. Grupo Técnico de Parceiros do Projeto Vida no Trânsito. Projeto Vida no Trânsito: avaliação das ações em cinco capitais brasileiras, 2011-2012. Epidemiol Serv Saúde 2013; 22(3):373-382.

24. Brasil. Ministério da Saúde (MS). Portaria 1.378, de 9 de julho de 2013. Regulamenta as responsabilidades e define diretrizes para execução e financiamento das ações de Vigilância em Saúde pela União, Estados, Distrito Federal e Municípios, relativos ao Sistema Nacional de Vigilância em Saúde e Sistema Nacional de Vigilância Sanitária. Diário Oficial da União 2013; 10 jul.

25. Malta DC, Lima MFF Leal MC, Morais Neto OL. Inquéritos Nacionais em Saúde: a experiência acumulada e a proposta do Inquérito Nacional de Saúde. Rev Bras Epidemiol 2008; 11(Supl. 1):159-167.

26. Brasil. Ministério da Saúde (MS). Secretaria de Vigilância em Saúde. VIGITEL Brasil 2012: Vigilância de fatores de risco e proteção para doenças crônicas por inquérito telefônico. Brasília: MS; 2013.

27. Instituto Brasileiro de Geografia e Estatística (IBGE). Coordenação de População e indicadores sociais. Pesquisa Nacional de Saúde do Escolar: 2012. Rio de Janeiro: IBGE; 2013.

28. Szwarcwald CL, Malta DC, Pereira CA, Vieira MLFP, Conde WL, Souza Júnior PRB, Damacena GN, Azevedo LO, Silva GA, Theme Filha MM, Lopes GS, Romero DE, Almeida WS, Monteiro CA. Pesquisa Nacional de Saúde no Brasil: concepção e metodologia de aplicação. Cien Saude Colet 2014; 19(2):333-342.

29. Brasil. Ministério da Saúde (MS). Secretaria de Vigilância em Saúde. Departamento de Doenças e Agravos não Transmissíveis e promoção da Saúde. Viva: Vigilância de Violências e Acidentes, 2009, 2010 e 2011. Brasília: MS; 2013.

30. Castro AM, Sperandio AMG, Gosch CS, Rocha DG, Cruz DKA, Malta DC, Albuqyerque GM, Zancan L, Westphal M, Neto OLM, Durán PRF, de Sá RF, Mendes R, Moysés ST, Marcondes W, organizadores. Curso de extensão para gestores do SUS. Promoção da saúde. Brasília: CEAD, FUB; 2010. 
31. Brasil. Ministério da Saúde (MS). Biblioteca Virtual em Saúde. Doenças Crônicas não Transmissíveis. Brasília: MS; 2010.

32. Brasil. Ministério da Saúde (MS). Guia Alimentar para a População Brasileira. Consulta pública no 04. Brasília: MS; 2014.

33. Brasil. Decreto da Presidência da República no 8.262, de 31 de maio de 2014 Altera o Decreto no 2.018 de $1^{\circ}$ de outubro de 1996, que regulamenta a Lei no 9.294 de 15 de julho de 1996. Diário Oficial da União 2014; 2 jun.

34. Malta DC, Dimech CPN, Mora L, Silva Junior JB. Balanço do primeiro ano da implantação do Plano de Ações Estratégicas para o enfrentamento das Doenças Crônicas Não Transmissíveis no Brasil, 2011 a 2022. Epidemiol. Serv. Saúde 2013; 22(1):171-178.

35. Jaime PC, Silva ACF, Lima AMC, Bortolini GA. Ações de alimentação e nutrição na atenção básica: a experiência de organização no Governo Brasileiro. Rev Nutr 2011;24(6):809-824.

36. Brasil. Termo de Compromisso no 4/2011. Termo de Compromisso que firmam entre si a União, por intermédio do Ministério da Saúde, e a Associação Brasileira das Indústrias de Alimentação (ABIA), a Associação Brasileira das Indústrias de Massas Alimentícias (ABIMA), a Associação Brasileira da Indústria de Trigo (ABITRIGO) e a Associação Brasileira de Panificação e Confeitaria (ABIP), com a finalidade de estabelecer metas nacionais para a redução do teor de sódio em alimentos processados no Brasil. Diário Oficial da União $2011 ; 26 \mathrm{dez}$.

37. Brasil. Ministério da Saúde (MS). Secretaria Executiva. Secretaria de Vigilância em Saúde. Glossário temático: promoção da saúde. Brasília: MS; 2012.

38. Brasil. Decreto de $1^{\circ}$ agosto de 2003. Cria a Comissão Nacional para Implementação da Convenção-Quadro para o Controle do Tabaco e de seus Protocolos. Diário Oficial da União 2003; 4 ago.

39. Amorim TC, Knuth A, Cruz DKA, Malta DC, Reis RS, Hallal PC. Descrição dos programas municipais de promoção da atividade física financiados pelo Ministério da Saúde. Rev Bras Ativ Fis e Saúde 2013; 18(1):6374.

40. Hallal PC, Knuth AG, Cruz DKA, Mendes MI, Malta DC. Prática de atividade física em adolescentes brasileiros. Cien Saude Colet 2010; 15(Supl. 2):3035-3042.

41. Pratt M, Brownson RC, Ramos LR, Malta DC, Hallal P, Reis RS, Parra DC, Simões EJ, Project GUIA. A model for understanding and promoting physical activity in Brazil and Latin America. J Phys Act Health 2010; 7(Supl. 2): S131-S134

42. Parra DC, McKenzie TL, Ribeiro IC, Ferreira Hino AA, Dreisinger M, Coniglio K, Munk M, Brownson RC, Pratt M, Hoehner CM, Simões EJ. Assessing physical activity in public spaces in Brazil using systematic observation methods. Am J Public Health 2010; 100(8):1420-1426. 
